\title{
Il progetto ArAl per un approccio relazionale all'insegnamento nell'area aritmetico-algebrica
}

\section{The ArAl project for a relational approach to teaching in the arithmetic-algebraic area}

\author{
Giancarlo Navarra \\ Insegnante ricercatore, già professore a contratto - Università di Modena e Reggio Emilia, Italia
}

Sunto / Nell'articolo si presenta il "Progetto ArAl, Percorsi nella didattica per favorire il pensiero prealgebrico" e si introduce l'ambito teorico all'interno del quale esso si colloca, l'early algebra: un campo relativamente nuovo di ricerca e pratica didattica che contrappone alla didattica tradizionale, che fa iniziare l'algebra verso i 13-14 anni di età, l'ipotesi che sia non solo possibile, ma anche opportuno, spostare questo inizio ai 6 anni, con un'attenzione crescente verso gli alunni "grandi" della scuola dell'infanzia ( 5 anni). Si riportano numerosi episodi di classe (alunni fra i 6 e i 15 anni) relativi in parte a classi “inesperte” e in parte a classi “esperte”. I commenti che li accompagnano illustrano i punti chiave del quadro teorico, la prospettiva linguistica che contraddistingue le radici epistemologiche del progetto, le differenze più significative fra l'early algebra e l'insegnamento tradizionale dell'aritmetica e dell'algebra.

Parole chiave: Early algebra; progetto ArAl; balbettio algebrico; pensiero relazionale; formazione dei docenti.

\begin{abstract}
The paper presents the "ArAl Project, Arithmetic pathways aimed at facilitating pre-algebraic thinking" and introduces early algebra, the theoretical framework within which it is placed. Early algebra is a relatively new field of research and teaching practice. Unlike the traditional teaching, where the study of algebra begins at the age of 13-14, our hypothesis is that not only is it possible, but also appropriate, to anticipate the teaching of algebra at the age of 6 , with an increasing attention towards the "great" pupils of the kindergarten ( 5 years old). Many class episodes (pupils aged 6 to 15) are reported, related partly to "inexperienced" classes (teachers are novices of the project) and partly to "expert" classes (skilled teachers who have been collaborating with the project for many years).
\end{abstract}

Keywords: Early algebra; ArAl Project; algebraic babbling; relational thinking; teacher training.

\section{Breve storia del progetto ArAl}

Il Progetto ArAl, Percorsi nell'aritmetica per favorire il pensiero prealgebrico nasce dai lavori condotti dai primi anni ' 80 dal GREM (Nucleo di ricerca in Educazione Matematica) operante presso il Dipartimento di matematica dell'Università di Modena e Reggio Emilia, sotto la direzione scientifica di Nicolina A. Malara'; si sviluppa grazie alle opportunità di distacco all'università offerte dal MIUR e alla collaborazione iniziale con una rete di 12 scuole distribuite in due regioni, il Veneto e l'Emilia Romagna. Negli anni '90 le premesse sociali e culturali del progetto si evolvono attraverso attività sperimentali svolte in compresenza da docenti e ricercatori in classi di scuola dell'infanzia, elementare e media proiettandosi sempre più verso la didattica dell'a- 
ritmetica e dell'algebra e collocandosi all'interno dell'area di ricerca denominata a livello internazionale early algebra. In questo contesto il progetto esplora possibilità e opportunità per lo sviluppo del pensiero prealgebrico fin dalla prima elementare, con una crescente attenzione verso gli alunni "grandi" della scuola dell'infanzia (5 anni). Nel 2001 il progetto ArAl vince il concorso nazionale SeT (Progetto per lo sviluppo Scientifico e Tecnologico) su più di 600 concorrenti (reti di scuole coordinate da istituti di ricerca pubblici o privati) e si aggiudica un finanziamento complessivo di 84 milioni di lire. Inizia la collaborazione con scuole o reti di scuole che negli anni successivi si amplia coinvolgendo quasi tutte le regioni italiane. Nel 2003 inizia la pubblicazione della Collana ArAl con la Pitagora Editrice di Bologna, composta attualmente (2018) dal testo introduttivo "Quadro teorico e Glossario" e da 13 Unità (AA.VV., 2003-2018). Dal 2001 il progetto è partner in progetti europei, partecipa a convegni nazionali e internazionali, corsi di formazione; la raccolta quasi completa delle pubblicazioni è consultabile nel sito del progetto. Tra il 2008 e il 2013 sviluppa in collaborazione con il GISCEL il progetto MTPAL (applicazione della Metodologia delle Trascrizioni Pluricommentate in Ambito Linguistico, par. 3.1) (Navarra, 2009; Deon \& Navarra, 2014; Navarra, 2016). Nel 2011 e nel 2018 il progetto ArAl viene invitato a partecipare a due importanti raccolte di articoli che illustrano il panorama internazionale delle principali ricerche sull'early algebra (Cusi, Malara \& Navarra, 2011; Malara \& Navarra, 2018). Nel 2015 viene aperta la quarta versione del sito Progetto ArAl; nello stesso anno viene attivato in Facebook il gruppo Progetto ArAl (attualmente di quasi 3000 membri). Nell'anno scolastico 2017/2018 il progetto ha collaborato con istituti e reti di scuole in Friuli-Venezia Giulia, Veneto, Emilia Romagna, Toscana, Liguria, Puglia. Nicolina Malara è la responsabile scientifica, Giancarlo Navarra è co-responsabile scientifico e coordinatore nazionale del progetto.

\section{2}

\section{Il progetto ArAl e l'early algebra}

L'ipotesi di base del progetto ArAl è che i principali ostacoli cognitivi nell'apprendimento dell'algebra derivino da un insegnamento dell'aritmetica troppo focalizzato, dalla prima elementare, su calcoli e risultati, che in questo modo influenza lo sviluppo del pensiero matematico generando difficoltà di carattere sia cognitivo che emozionale. Si ritiene cioè che la consueta didattica dell'aritmetica induca negli alunni un imprinting che condiziona, o addirittura impedisce, nel corso degli studi - in modi spesso anonimi, sotterranei, difficilmente individuabili da parte dei docenti - la costruzione di competenze stabili e significative nell'ambito matematico. La ricerca a livello internazionale, a partire dai primi anni ' 80 , mette in evidenza come studenti introdotti all'algebra solo verso la fine della scuola media mostrino un'interpretazione inadeguata del segno uguale, manifestino difficoltà con la comprensione delle lettere soprattutto quando hanno il significato di variabile, trovino ostacoli nel produrre o nell'interpretare un'espressione algebrica come risposta valida alla soluzione di un problema, sbaglino nel risolvere equazioni con una presenza di lettere in entrambi i lati del segno "=" (questi e altri aspetti vengono trattati ampiamente in Carraher \& Schliemann, 2018). Al contrario, uno spostamento dell'attenzione dalla ricerca dei risultati alla riflessione sui significati è il fattore decisivo per portare con gradualità alunni impegnati nello studio dell'aritmetica a cogliere il generale anche 
operando su casi particolari e per avvicinarli alla rappresentazione simbolica di relazioni e proprietà, gettando così le basi per il consolidarsi del pensiero relazionale e quindi per la comprensione del linguaggio algebrico.

Lo stesso David Carraher (Carraher, Schliemann \& Schwartz, 2007), ricercatore impegnato da tempo in questo campo, titola significativamente un capitolo del suo libro Algebra in the early grades: Early Algebra Is Not the Same as Algebra Early. Per comprendere il gioco di parole, bisogna capire cosa si intenda per early algebra. Si potrebbe tradurre con "approccio precoce all'algebra", ma I'aggettivo precoce non convince perché riconduce ad una ipotetica norma che sancirebbe un momento giusto per la sua introduzione. Nemmeno "approccio anticipato all'algebra" va bene perché anche anticipato ha in sé una valenza di prima del dovuto che si potrebbe interpretare come tentativo azzardato. Riteniamo che traduzioni adeguate siano "algebra degli inizi" o, per gli alunni di 5-7 anni, "la prima algebra", con una connotazione quasi affettuosa e una sfumatura marcatamente ludica, del genere "il mio primo libro", "la mia prima bambola"2. Quindi, "algebra degli inizi" non significa iniziare prima l'algebra ma percorrere l'aritmetica in modi tali da favorire lo sviluppo del pensiero prealgebrico, scoprire quindi il volto algebrico di ciò che spesso è visto solo come aritmetica (per una breve storia dell'early algebra e della sua evoluzione, v. Kieran, Pang, Schifter \& Ng, 2016).

Nell'ottica del progetto ArAl, questo sviluppo avviene gradualmente lungo la progressione temporale illustrata in Figura 1:

Figura 1

Progressione e intrecci

fra l'early algebra e

lo sviluppo del pensiero algebrico.

\begin{tabular}{|c|c|c|c|c|c|c|c|c|c|c|c|c|c|}
\hline \multicolumn{14}{|c|}{ Early Algebra (EA) } \\
\hline 5 & 6 & 7 & 8 & 9 & 10 & 11 & 12 & 13 & 14 & 15 & 16 & 17 & 18 \\
\hline \multicolumn{6}{|c|}{ EA } & \multicolumn{4}{|c|}{ EA-PA } & \multicolumn{4}{|c|}{$\mathrm{PA}$} \\
\hline & & & & & & \multicolumn{8}{|c|}{ Pensiero Algebrico (PA) } \\
\hline
\end{tabular}

La fase dell'early algebra (EA) riguarda gli alunni fra i 5 e i 14 anni, lo sviluppo del pensiero algebrico (PA) quelli dagli 11 anni in poi; il periodo scolastico fra gli 11 e i 14 anni è molto delicato perché è quello in cui avviene il passaggio graduale tra le due fasi (EA-PA). La differenza profonda tra la didattica tradizionale dell'aritmetica e dell'algebra e la prospettiva rappresentata dall'early algebra è quindi che mentre la prima vede l'algebra introdotta successivamente all'aritmetica a partire dalla terza media, la seconda sostiene una didattica costruita, sin dall'inizio, sull'intreccio fra le due discipline. Sviluppare con gradualità il pensiero algebrico in tale contesto significa costruire negli studenti l'attitudine verso l'esplorazione di regolarità, relazioni e proprietà, la capacità di riflettere su di esse e rappresentarle prima attraverso il linguaggio naturale e poi attraverso quello algebrico, le competenze nel concepire e nell'usare, da parte degli alunni più grandi, il linguaggio algebrico come strumento di pensiero (questa panoramica viene illustrata con esempi di attività e analisi di episodi di classe in Carpenter, Frank \& Levi, 2003).

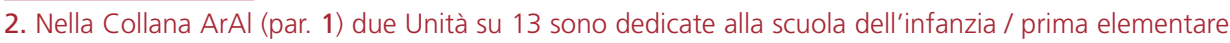
hanno l'obiettivo di mostrare come la prima costituisca, a tutti gli effetti, il primo gradino della scala che vede aritmetica e algebra intrecciate sin dall'inizio del percorso educativo: l'Unità 10, Qual è il colore della sedia? Successioni modulari e forme embrionali di generalizzazione e I'Unità 13, La Matematochetta. Gioco dell'aggiungere e del togliere in preparazione all'addizione e alla sottrazione. 
In questa concezione è fondamentale il ruolo svolto nell'insegnamento della matematica dal linguaggio o, meglio, dai linguaggi, a cominciare da quello naturale. È da qui che nasce il costrutto che abbiamo chiamato balbettio algebrico, un processo attraverso il quale il bambino, in analogia con quanto avviene nell'apprendimento del linguaggio naturale, si appropria poco alla volta, sin dai primi approcci all'aritmetica, dei significanti, dei significati e delle regole che supportano il linguaggio algebrico, in un ambiente educativo tollerante verso i tentativi, la creatività, l'imitazione, gli errori che contraddistinguono l'apprendimento di un qualsiasi linguaggio. Questo percorso non è semplice per l'insegnante perché, come vedremo, esso costituisce una sorta di rivoluzione copernicana nelle sue conoscenze e nella sua didattica dell'aritmetica e dell'algebra. Nel progetto ArAl sono stati concepiti numerosi strumenti per aiutarlo a padroneggiare il balbettio algebrico e le modalità per favorirlo. II cuore teorico di tali strumenti è costituito dal sistema dei Glossari: sono quattro, per un complesso di più di 200 termini o costrutti, collegati fra loro da una fitta rete di link. L'esplorazione dei glossari è una lenta avventura intellettuale dell'insegnante, che costruisce i suoi personali itinerari scegliendo di volta in volta i link che attirano maggiormente la sua attenzione. Qualsiasi percorso egli compia conduce comunque, attraverso ampliamenti e approfondimenti successivi, ad una visione relazionale dei termini chiave che compongono i glossari, e quindi ad una comprensione della teoria.

Quadro teorico e Glossari aiutano a capire come la costruzione di competenze matematiche in una prospettiva algebrica (Figura 2) poggi, sin dalle fasi iniziali, su solide basi di carattere linguistico, supportate a loro volta da presupposti di natura sociale e psicologica e da una serie di concetti di tipo generale.

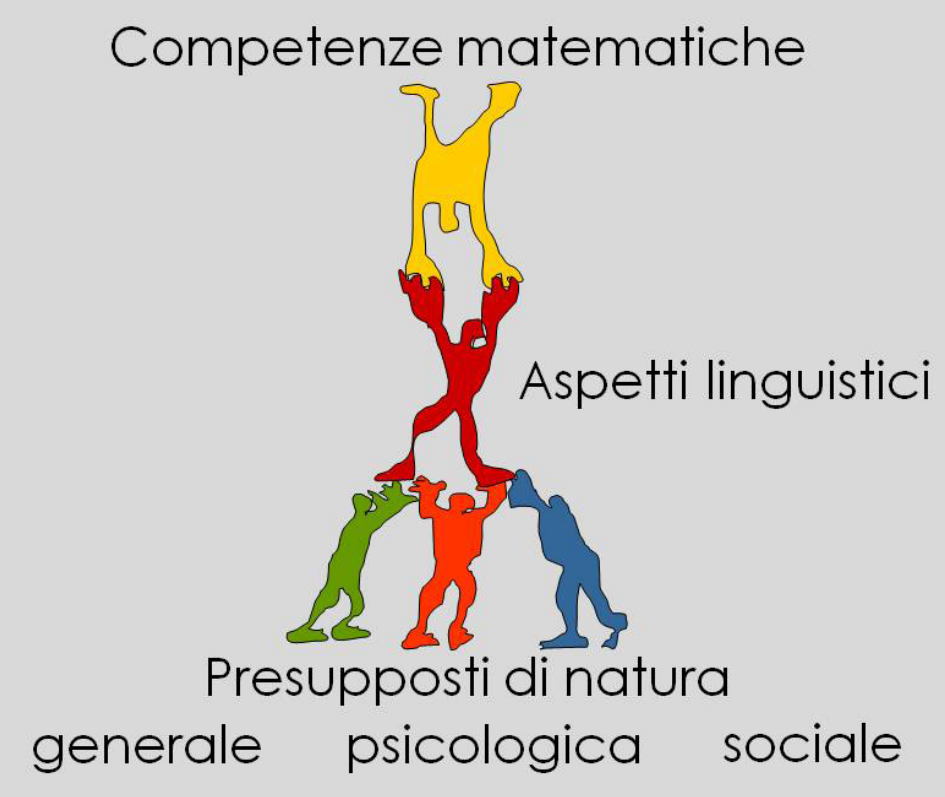

Prima di proseguire nell'analisi dei punti chiave che possono favorire o ostacolare lo sviluppo del pensiero aritmetico verso quello algebrico, presentiamo una sintesi dei principali strumenti operativi del progetto ArAl che supportano i docenti nella comprensione delle interazioni fra prassi e teoria. 


\title{
3 Principali strumenti del progetto ArAl per favorire le competenze dei docenti nell'ambito dell'early algebra
}

\author{
3.1 La Metodologia delle Trascrizioni Pluricommentate (MTP): \\ favorisce la riflessione sull'attività di classe dal punto di vista matematico, linguistico, \\ metodologico attraverso trascrizioni (chiamate diari) di audio o video registrazioni \\ di episodi. Ogni autore, dopo aver commentato il diario, lo invia al suo e-tutor che \\ lo commenta a sua volta, spesso invitando altri ricercatori o docenti esperti a fare \\ altrettanto; a questo punto il diario viene ritrasmesso all'autore e ad altri docenti ap- \\ partenenti ad istituti che collaborano con il progetto ArAl. I diari si prefigurano così \\ come strumenti di mediazione fra teoria e prassi su più piani: \\ - diagnostico, forniscono un quadro delle competenze e della sensibilità dell'inse- \\ gnante (il concetto di "sensibilità" viene illustrato nel par. 6.1); \\ - formativo, consentono all'insegnante di riflettere sulla qualità della sua azione \\ didattica; \\ - valutativo, danno a insegnanti e ricercatori elementi per valutare le ricadute della \\ teoria sulla pratica d'aula; \\ - sociale, favoriscono, mediante la loro diffusione, la condivisione dei saperi in \\ gioco.
}

\subsection{Gli interventi di un esperto ArAl in classe:}

in genere è un ricercatore-formatore. Spesso questa figura coincide con quella dell'e-tutor prevista dalla MTP; svolgono questo ruolo anche insegnanti che si sono dotati di competenze riconosciute nel corso di collaborazioni pluriennali con il progetto ArAl. In occasione degli incontri di formazione (par. 3.3) l'esperto gestisce delle attività in classi dall'infanzia alla terza media (della durata di una normale lezione) alla presenza dell'insegnante di classe e, spesso, di altri osservatori (colleghi dello stesso o di altri istituti, ricercatori, tirocinanti, laureandi, qualche volta genitori). L'esperto nei suoi interventi opera esplicitamente in funzione dei docenti che assistono, svolgendo il ruolo di modello e mostrando come sia possibile stimolare negli alunni atteggiamenti che favoriscano lo sviluppo del balbettio algebrico attraverso una didattica impostata in chiave socio-costruttivista.

\subsection{Gli incontri di formazione:}

per la maggior parte si svolgono all'interno delle collaborazioni pluriennali fra istituti o reti e il progetto ArAl; durante il loro svolgimento si approfondiscono e si ampliano assieme agli insegnanti, anche in forme laboratoriali, aspetti matematici, linguistici o metodologici del progetto e dell'early algebra, si organizzano riflessioni comuni sui diari (par. 3.1) e sugli spunti derivanti dagli interventi degli esperti nelle classi (par. 3.2), configurando così un sistema formativo integrato. ${ }^{3}$ Altri incontri vengono promossi da enti, associazioni o istituti secondo modalità organizzative più tradizionali.

3. Ogni anno scolastico questi incontri sono almeno tre, di due giorni consecutivi ciascuno; al mattino l'esperto svolge interventi in classi dalla scuola dell'infanzia alla terza media; il pomeriggio incontra i docenti I tre incontri si svolgono a distanza di tempo di circa due mesi l'uno dall'altro; durante questi intervalli docenti impegnati nella collaborazione redigono diari MTP. 


\section{$4 \begin{aligned} & \text { I punti chiave del passaggio dalle concezioni più diffuse nella di- } \\ & \text { dattica dell'aritmetica e dell'algebra all'early algebra }\end{aligned}$}

Ogni insegnante è plasmato dal suo retroterra culturale definito da conoscenze, convinzioni, esperienze, abitudini, stereotipi. È decisiva in questo quadro la sua formazione all'origine, maturata nell'ambiente familiare prima e negli anni della scuola poi, sino al compimento del ciclo di studi superiori: un imprinting che determina il rapporto profondo che ha stabilito - nel bene e nel male - con la matematica, e che influisce sul suo insegnamento.

Analizzerò di seguito alcuni concetti radicati nell'insegnamento tradizionale dell'aritmetica collegandoli - spesso contrapponendoli - ai corrispondenti costrutti teorici del progetto ArAl. Farò vedere come e perché essi possano rappresentare degli ostacoli allo sviluppo del pensiero algebrico e come questi ostacoli possano essere affrontati per indurre, negli stessi docenti prima e negli alunni poi, concezioni favorevoli alla promozione del pensiero algebrico sin dalla prima elementare. Li illustrerò attraverso l'analisi di episodi di classe relativi alla scuola elementare (P1 sta per Prima Elementare e così via sino a P5) e alla scuola media (M1-M4) ${ }^{4}$, estrapolati da diari MTP (par. 3.1). ${ }^{5}$

Dividerò gli episodi in due gruppi, relativi rispettivamente a: A. classi "inesperte" e B. classi "esperte" nell'ambito dell'early algebra. Definisco classi "inesperte" quelle guidate da insegnanti all'inizio di un rapporto di collaborazione con il progetto ArAl, che quindi possiedono prevalentemente modelli di pensiero condizionati dal retroterra culturale standard descritto all'inizio di questo paragrafo. Definisco al contrario classi "esperte" quelle il cui docente, attraverso più anni di collaborazione con il progetto ArAl, abbia maturato una conoscenza sempre più ampia e approfondita dei suoi costrutti e delle prospettive aperte dall'early algebra in termini di contenuti e di metodi.

\subsection{Episodi relativi a classi "inesperte" \\ 4.1.1 ( $\mathrm{P} 1$, verso la fine dell'anno scolastico)}

Alla LIM si è giunti alla scrittura $11+7=18$. Si propone, accanto ad essa, $18=$ $11+7$ e si chiede alla classe se vada bene. La risposta è un vibrato e collettivo «No!». Si invita Gaia a spiegare il perché del suo No. Gaia rimane incerta e poi dice che sì, «11 più 7 fa 18, ma 18 uguale a 11 più 7 non va bene com'è scritta perché i numeri sono scambiati».

La classe di Gaia non accetta $18=11+7$ perché non capisce come sia possibile che il risultato stia a sinistra e l'operazione a destra. È abituata ad operare sulle scritture matematiche, non a riflettere su di esse. In questa concezione l'uguale possiede il significato di operatore direzionale, con una connotazione spazio-temporale: prepa-

4. L'unico episodio indicato con M4 si riferisce ad una classe prima di scuola secondaria di secondo grado, detta anche scuola superiore in Italia, che corrisponde al quarto anno di scuola media in Ticino.

5. Dal 2002, anno in cui è iniziata ufficialmente la pratica dei diari, ad oggi (2018) sono stati redatti quasi 300 diari, ognuno dei quali è corredato mediamente da 30-40 fra commenti e meta-commenti. Per un totale di più di 4500 commenti. 
ra il finale di una storia che inizia a sinistra con dei calcoli e si conclude a destra con un risultato. II simbolo "=", per come viene concepito da questi alunni, è veicolo di significati poveri; non possiede un significato relazionale, nel senso che non indica l'equivalenza fra due quantità. È la pura traduzione della voce verbale "fa": "11 più 7 fa 18" (e 18 "non fa" 11 più 7).

\subsection{2 (P2, inizio anno scolastico)}

Si chiede a Rita di scrivere "36 - 24"; I'alunna scrive "36 - 24 =" e chiede «Devo trovare quanto fa?» Di fronte al silenzio dell'insegnante dopo l'uguale scrive 12 .

Rita esprime un disorientamento analogo a quello manifestato da Gaia nell'esempio precedente: "36 - 24" anche per lei è un'operazione, cioè una scrittura "in attesa". L'invito "Scrivi 36 - 24" viene interpretata come "Calcola 36 - 24". Di conseguenza Rita inserisce il simbolo " $=$ " per preparare il risultato, perché la scrittura $36-24$, da sola, è qualcosa di provvisorio e come tale è un oggetto povero di significato: Rita è abituata al fatto che, prima o poi, le verrà chiesto "Quanto fa?". Dei ricercatori hanno chiamato il bisogno di inserire comunque il segno "=" sindrome da mancanza di risultato.

\subsection{3 (P3, inizio anno scolastico)}

Si propone, nel corso di un'attività sul significato attribuito al segno " $=$ ", la frase:

$$
14+21=39 .
$$

Dopo un po' si sentono delle voci «No!», «Non va bene!», «È sbagliato!». Si chiede come si potrebbero tradurre in linguaggio matematico queste prese di posizione. Qualcuno propone di correggere a destra il risultato e scrivere 35. Si chiede in quale altro modo si potrebbe fare. Qualche alunno suggerisce di modificare la parte sinistra in $18+21$, altri in $14+25$.

Gli alunni intervengono sull'operazione o sul risultato perché sono abituati a correggere degli errori nei calcoli. Non sono stati educati a vedere ai lati dell'uguale le rappresentazioni di due numeri (il concetto verrà approfondito nel par. 5.c), e quindi a riflettere sulla loro uguaglianza esprimendo ad un livello metacognitivo l'esito del confronto, prima in linguaggio naturale, ad esempio «Non è vero che $14+21$ è uguale a 39» oppure «14 + 21 non è uguale a $39 »$, e poi con la sua traduzione in linguaggio matematico: $14+21 \neq 39$. In questo modo mostrerebbero familiarità anche con il simbolo " $\neq$ ", raramente introdotto nella scuola elementare proprio perché non viene favorita la riflessione sulle scritture ma l'esecuzione di un calcolo. L'uguale mantiene quindi nei primi anni di scuola un significato procedurale. Un contratto didattico che invece promuova l'analisi delle scritture per confrontare il loro valore condurrebbe gli alunni a proporre di rappresentare la stessa relazione anche come $14+21<39$, puntualizzando così ulteriormente la disuguaglianza fra i due numeri. 


\subsection{4 ( $\mathrm{P} 4$, anno scolastico avanzato)}

Si presenta la scrittura $11+4=7+8$. Molti alunni dicono che va bene ma altri non sono d'accordo. II clima generale è poco convinto. La discussione fa emergere un conflitto di percezioni che porta ad una instabilità concettuale: la scrittura sì, andrebbe bene, ma...

Il conflitto che si manifesta è strettamente legato agli episodi precedenti; lo illustriamo evidenziando con coloriture diverse le due diverse percezioni che si intersecano generando dei cortocircuiti logici:
(a) $11+4=7+8$
(b) $11+4=7+8$

In (a) è evidenziata in giallo l'interpretazione spontanea da parte di un alunno: opacizza " +8 ", percepisce " $11+4=7$ " come "operazione = risultato", ma non gli suona bene perché 7 non è il risultato corretto. Una conferma di tale ambiguità interpretativa è data dal fatto che una frase come " $11+4=15+8$ " viene sentita invece da molti come corretta perché 15 è percepito come risultato di $11+4$, anche se poi c'è quel " +8 " che non si sa bene come decifrare. Una tale interpretazione rientra nella categoria che abbiamo chiamato Lettura partigiana del testo. Com'è evidenziato in celeste in (b), è necessario che gli alunni spostino l'attenzione verso la relazione che collega " $11+4 "$ e " $7+8$ ", e quindi verso il simbolo " $=$ " che la rappresenta, e vedano la frase " $11+4=7+8$ " come oggetto unico, cioè come uguaglianza fra due rappresentazioni dello stesso numero (15). Devono superare il punto di vista procedurale - operativo, fattuale - e dotarsi di uno relazionale. È questa prospettiva che permette di spostare l'attività su un livello metacognitivo, portando gli alunni a valutare la correttezza dell'uguaglianza attraverso il confronto fra le due scritture ai lati dell'uguale. Ė necessario quindi costruire il passaggio dal significato operativo-aritmetico dell'uguale come operatore direzionale a quello relazionale-algebrico dell'uguale come indicatore di equivalenza fra due quantità.

\subsection{5 (M2, inizio anno scolastico)}

L'insegnante propone agli studenti l'esplorazione delle piramidi di numeri. ${ }^{6}$ Presenta tre minipiramidi:
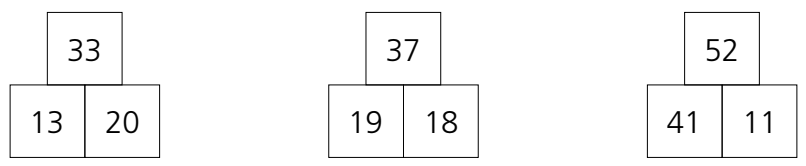

Chiede di individuare la "regola delle piramidi" e di verbalizzarla. La classe perviene a questa definizione «Per trovare il numero in alto bisogna sommare i due numeri nei mattoni in basso». Opportunamente guidati, gli alunni giungono ad una nuova enunciazione che dice non come si trova ma cos'è il numero in alto: «In una minipiramide il numero in alto è la somma dei numeri 
scritti nei mattoni alla base». Si propone una minipiramide con alla base 25 e 67 e si chiede di scrivere nel mattone in alto in linguaggio matematico la traduzione di quest'ultima regola. Gli studenti scrivono " $25+67$ " e dopo un veloce calcolo mentale aggiungono " $=92 "$.

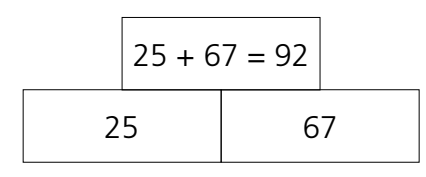

Si stupiscono quando si chiede perché secondo loro sia necessario aggiungere "=92". Si guardano l'un l'altro e spiegano «Ma è ovvio, che domanda è? Abbiamo scritto il risultato».

Il condizionamento visto negli episodi precedenti grava anche su questi studenti (con sette anni di scuola alle spalle): per loro $25+67$ è un'operazione e deve portare ad un risultato che non può che essere esplicitato, e infatti aggiungono " $=92$ ". $25+$ 67 , da solo, non significa nulla perché è loro estraneo il concetto di scrittura matematica come rappresentazione di un numero. Una scrittura, per così dire, ontologicamente compiuta.

Per quanto concerne le due diverse definizioni, la prima dice ciò che bisogna fare, esprime cioè un punto di vista procedurale, che è quello che porta ad aggiungere "=92"; la seconda spiega cos'è il numero in alto, ed enuncia la struttura additiva della sua rappresentazione. Ma siccome la classe incontra per la prima volta la dualità procedurale / relazionale e non ha confidenza con il termine "rappresentare" (e quindi con la dualità rappresentazione canonica / non canonica di un numero, par. 5.c) continua a chiamare la scrittura $25+67$ operazione; il termine "somma" usato nella frase " 25 + 67 è la somma fra 25 e 67" continua ad essere visto come prodotto, non come processo. Ci troviamo in presenza di quello che è stato chiamato da Malara (1994) "dilemma processo-oggetto":

«Come sottolineato da A. Sfard (1991), la costruzione dei concetti algebrici si sviluppa per successivi livelli di astrazione ma prevalentemente attraverso processi computazionali, pertanto ogni oggetto matematico viene a riassumere in sé due aspetti complementari, quello di processo e quello di oggetto, come due facce di una stessa moneta. Nel primo aspetto prevale il punto di vista operazionale che è dinamico e sequenziale, nel secondo l'oggetto è visto come entità statica e fuori dal tempo e si considera da un punto di vista strutturale (si pensi ad esempio all'equazione vista come procedimento di codifica delle relazioni espresse da un problema ed all'equazione vista come oggetto di studio in sé). Lo sviluppo del pensiero algebrico, e più in generale della matematica, è caratterizzato da questo passaggio dal procedurale allo strutturale».

(Malara, 1994, p. 68)

\subsection{6 (M3, seconda metà anno scolastico)}

Lara ha imparato con la sua insegnante che $a^{2}-b^{2}$ è "la differenza fra due quadrati". Le si chiede «Cos'è $5^{2}-3^{2}$ ?» e Lara risponde «16». Non si sogna di dire $« 5^{2}-3^{2}$ è la differenza fra il quadrato di 5 e il quadrato di $3 »$. 
L'abitudine ad operare in senso procedurale ostacola la concettualizzazione di cosa siano gli oggetti su cui si opera e delle loro proprietà. Questo spiega la mancata interpretazione da parte di Lara di $5^{2}-3^{2}$ in termini di "differenza di quadrati" e la tendenza da parte sua a vedere questa scrittura come un insieme di operazioni da svolgere secondo un determinato ordine che lei mostra di conoscere giungendo al risultato corretto 16. Portare l'attenzione di alunni giovani verso le rappresentazioni di un numero ha ricadute importantissime su altri temi, come ad esempio sulla soluzione dei problemi (v. la dualità rappresentare / risolvere nel par. 6.1.1. e nel par. § 7).

\subsection{7 (M4, inizio dell'anno scolastico)}

In una batteria di prove d'ingresso (in tutto 12 batterie centrate sulle competenze legate al tradurre frasi dal linguaggio naturale a quello algebrico e viceversa, all'esprimere relazioni fra dati, all'argomentare) organizzate da un gruppo di docenti della scuola superiore coordinati da un'esperta ArAl viene proposto il seguente quesito:

Traduci in linguaggio matematico: "Il successivo di $r$ ".

Gli studenti scrivono nei commenti alle prove (vi erano nelle schede spazi appositi in questo senso) di aver incontrato difficoltà in questa traduzione perché non ricordano né il significato di "successivo di un numero" né il senso della precisazione "di un numero naturale"; in genere lasciano in bianco la risposta; una traduzione che compare più volte è: $r+r$.

Gli studenti, per capire cosa significa tradurre una frase, dovrebbero saperla interpretare, cioè attribuire un significato alla sua struttura e quindi alle relazioni fra i suoi termini. Se sono abituati - solo o prevalentemente - alla logica del fare non sono educati a ragionare sul senso delle scritture, siano esse in linguaggio naturale oppure matematico. Non sono quindi abituati a concepire due frasi come traduzioni I'una dell'altra. Per fare questo, dovrebbero essere guidati, sin dalla prima elementare, a vedere nella matematica un nuovo linguaggio, dotato - come qualsiasi linguaggio - di una semantica e una sintassi; un linguaggio per comunicare, attraverso il quale costruire e interpretare frasi e produrre parafrasi sia in linguaggio naturale che matematico; le parafrasi nel linguaggio naturale diventano mediatori verso la loro traduzione in linguaggio matematico (e viceversa). Nell'episodio, durante la discussione sugli esiti delle prove, gli studenti definiscono il termine "successivo" attraverso una circonlocuzione del tipo: «Che viene dopo» oppure portando un esempio: «Dopo 24 c'è 25». La traducibilità della prima frase è resa impossibile dall'opacità - in termini di traghetto semantico verso il linguaggio matematico - delle parole "che viene dopo". Un'attenzione proiettata verso gli aspetti della matematica come linguaggio e le mutue relazioni fra linguaggio naturale e linguaggio matematico guiderebbe a formulare parafrasi complete (in termini di soggetto, predicato, complemento) e funzionali all'individuazione della traduzione. Ad esempio, in questo caso, la frase «॥ successivo di un numero naturale è la somma fra il numero stesso e $1 »$ porterebbe a concludere che, poiché il successivo di 24 è esprimibile in forma non canonica (par. 5.c) come $24+1$, il successivo di $r$ sarà rappresentabile (si può parlare di traduzione letterale) come $r+1$. 


\section{Sintesi dei punti chiave che emergono nei primi sette episodi}

Prima di passare agli episodi del gruppo B (classi esperte), tiriamo le fila dei discorsi fatti sinora concentrandoci sui principali aspetti emersi.

a. L'argomentazione. Gli atteggiamenti di tutti gli alunni citati mostrano i depositi di ciò che resta, nel profondo, delle conoscenze acquisite dall'inizio della loro educazione matematica, legate soprattutto al fare e al calcolare. Essi sono abituati ad operare sulle scritture matematiche, non ad argomentare su di esse. Le spiegazioni, come si è visto, molto spesso non sono semplici, ma proprio per questo bisogna concordare con la classe un contratto didattico che superi gli "iceberg linguistici", quelle situazioni cioè nelle quali, per un tacito accordo fra alunni e docente, sono ritenute sufficienti poche (spesso pochissime) parole per esprimere la parte sommersa. Solo quando l'argomentazione diventa una pratica sociale condivisa nella classe - e quindi un valore comune - essa può esprimere il ruolo di traghetto verso la generalizzazione, e gli studenti possono essere gradualmente resi consapevoli del ruolo che essa gioca nello sviluppo della loro capacità di riflettere sulle proprie conoscenze matematiche (Cusi \& Navarra, 2012).

b. L'uguale nell'accezione procedurale e in quella relazionale. Emerge, con sfumature diverse, il condizionamento indotto dall'idea tradizionale dell'aritmetica - insistiamo su questo aspetto cruciale - del fare. Un'operazione fa un risultato: ciò che sta a sinistra e ciò che sta a destra dell'uguale sono cose ontologicamente diverse; I'uguale non attira l'attenzione degli alunni perché sono concentrati sui calcoli. Quando si incontra l'algebra, invece, I'uguale assume un significato del tutto diverso, di tipo relazionale: indica l'equivalenza e, per simmetria, I'interscambiabilità di due rappresentazioni di una stessa quantità (ma la proprietà simmetrica dell'uguaglianza non viene quasi mai affrontata nei primi otto anni di scuola). Gli studenti della scuola media degli episodi 4.1 .6 e 4.1.7. si muovono in modo inconsapevole (perché nessuno li ha "avvertiti" del cambiamento) in un universo concettuale del tutto differente da quello aritmetico al quale sono abituati. Si adeguano, ma nella maggior parte dei casi rimangono poveri sul piano del controllo dei significati degli oggetti e dei processi. Se per loro "somma" è il nome del risultato di un'addizione, come interpretano il concetto di "somma tra variabili"? Non sono stati provvisti dei necessari traghetti semantici: se fossero abituati ad interpretare, ad esempio, $5+8$, come somma fra 5 e 8 , sarebbe più semplice per loro concepire come somma la scrittura $a+b$ (v. par. 6.1.1.iv) perché presenterebbe un'analogia strutturale con $5+8$, analogia che manca nel confronto con la rappresentazione canonica 13.

c. La rappresentazione. Alcuni fra i principali traghetti semantici sono le dualità forma canonica / forma non canonica di un numero, processo / prodotto, trasparente / opaco: la forma non canonica di un numero (ad esempio $5+8$ ) è trasparente in termini di significato perché ha un senso in relazione al contesto e al processo soggiacente, ed esprime (in questo caso) una rappresentazione additiva del numero 13. Viceversa, la forma canonica di un numero (ad esempio 13) è opaca in termini di significato perché non dice nulla rispetto al processo soggiacente.

Questa difficoltà interpretativa è diffusa anche in studenti della scuola superiore e in insegnanti in formazione. Di fronte alla domanda «Cos'è $[(11+7): 9]^{2} \times 3$ ?» rispondono: «Sono operazioni», «Sono calcoli», «È un'espressione da risolvere», 
«Devo trovare quanto fa», "Prima si trova la somma, poi si divide... »: I'imprinting del fare. Per recuperare il significato di numero - o, se si preferisce, di rappresentazione di un numero - è necessario introdurre gli alunni ai concetti di forma (o rappresentazione) canonica / non canonica. A questo scopo ricorriamo, a cominciare dalla prima elementare, alla strategia di trascrivere alla LIM, accanto al nome di un alunno, delle informazioni che lo riguardano: Laura, Sorella di Aldo, Amica del cuore di Julia, Padrona del cane Flic, eccetera. Laura è il nome proprio e le altre sono rappresentazioni che la definiscono offrendo informazioni che il nome non dà. Per inumeri-spieghiamo-la situazione è analoga: ogni numero può essere rappresentato in infiniti modi diversi. Per esempio "dodici" può essere rappresentato con la scrittura "12", ossia in forma canonica, oppure mediante delle espressioni equivalenti come " $3 \times 4$ ", " $(2+2) \times 3$ ", " $36 / 3$ ", " $10+2$ ", " $3 \times 2$ ", sue forme non canoniche, ognuna delle quali ha un senso legato al processo che la caratterizza (triplo di 4, quadruplo di 3, rapporto fra 36 e 3, somma fra 10 e 2, ecc.). Saper riconoscere e interpretare sin dai primi anni di scuola le forme non canoniche di un numero costruisce la base per comprendere scritture come $a b,-4 p, x^{2} y, k / 3$. Essere guidati a sviluppare il pensiero relazionale significa saper interpretare e definire $[(11+7): 9]^{2} \times 3$ ad esempio come "Il quadrato di un quoziente moltiplicato per 3 "; ma potrebbe anche essere "Il prodotto fra un quadrato e 3 ", oppure "Il triplo del quadrato di un quoziente", o un più evoluto "Un multiplo di 3". Rimanere confinati in un ambito procedurale significa per lo studente limitarsi ad una descrizione dei calcoli: «Sommo 11 e 7, poi divido per 9, poi faccio il quadrato e infine moltiplico per 3». Per far sì che uno studente sia in grado di costruire definizioni relazionali è necessario che egli venga guidato sin dai primi anni di scuola a comprendere la struttura degli oggetti matematici su cui dovrà operare. Essere in grado di produrre e interpretare parafrasi è una delle principali premesse ad una buona competenza linguistica (in senso lato), perché in questo modo si affina anche il controllo dei termini appropriati.

\section{Episodi relativi a classi esperte}

Gli episodi che presento ora sono tratti da diari di attività svolte in classi del secondo biennio della scuola elementare (quarta e quinta) che sin dalla prima operano in ambiente early algebra, e hanno avuto quindi il tempo di maturare con gradualità la qualità del loro balbettio algebrico. II livello dell'esperienza è favorito da molti fattori che riguardano l'insegnante: la durata della collaborazione con il progetto ArAl, I'intensità dello studio dei suoi contenuti, la disponibilità a mettersi in gioco anche redigendo dei diari (v. par. 3.1), la coerenza con cui in classe sa mantenere l'equilibrio fra teoria e prassi, la maturazione di una sensibilità che gli consenta di assumere, di fronte alle micro-situazioni che si presentano di continuo nella classe, opportune micro-decisioni coerenti con il percorso che ha scelto di seguire. John Mason descrive così questo concetto:

«Ogni professionista, indipendentemente dall'ambito in cui opera, desidera saper cogliere le possibilità, essere sensibile alle situazioni e rispondere in modo appropriato. Ma ciò che si considera appropriato dipende da ciò a cui 
si attribuisce valore, che dipende sua volta da ciò che si è capaci di notare. (...) [Nel caso dell'insegnante] notare ciò che gli alunni fanno o come rispondono, valutare ciò che dicono anche contro le proprie aspettative e i propri criteri di valutazione e considerare ciò che potrebbe essere detto o fatto in seguito. È sin troppo ovvio dire che non si può intervenire su ciò che non si nota; non si può scegliere di fare qualcosa se non si ravvisa l'opportunità di farlo».

(Mason, 2002, p.7, traduzione dell'autore)

I primi due episodi che illustrerò riguardano due quarte; sono centrati su situazioni problematiche (da noi considerevolmente ampliate rispetto a spunti illustrati in Steinweg, Akinwunmi \& Lenz, 2018) facenti parte dell'attività che abbiamo chiamato "Scatole \& Biglie", avviata nel 2017 in classi dalla scuola dell'infanzia alla terza media. L'ambiente riguarda due amici che giocano con biglie che possono tenere sciolte (e quindi visibili), oppure dentro scatole chiuse. L'attività, che si sviluppa con modalità concrete per i più piccoli e attraverso rappresentazioni iconiche per i più grandi, ha due regole: (1) in ogni situazione presentata i due bambini hanno un numero uguale di biglie; (2a) scatole dello stesso colore contengono lo stesso numero di biglie e, viceversa, (2b) scatole di colore diverso contengono numeri diversi di biglie. ${ }^{7}$

Ogni situazione è accompagnata da quattro consegne (talvolta sono cinque, come in 6.1.1., situazione 3):

A. Descrivi la situazione che vedi;

B. Quante biglie contiene/contengono la/e scatola/e? Argomenta la tua risposta;

C. Rappresenta la situazione in linguaggio naturale;

D. (per gli alunni più grandi) Rappresenta la situazione in linguaggio matematico per Brioshi (questo personaggio viene illustrato nel par. 6.1.1.v.)

In alcune classi "esperte" gli insegnanti hanno condotto l'attività intrecciandola con quella della bilancia a piatti ${ }^{8}$, utilizzando entrambe come approccio all'equazione.

\subsection{1 (P4, fine dell'anno scolastico)}

L'insegnante propone, una alla volta, tre situazioni; si eseguono collettivamente le prime tre consegne, la quarta è il frutto di attività individuali i cui esiti vengono poi confrontati, discussi ed eventualmente migliorati collettivamente prima di essere inviati Brioshi. Alcuni esempi di protocolli nella pagina successiva:

\footnotetext{
7. Agli alunni più grandi (dalla quarta-quinta elementare) si chiede di ipotizzare cosa succede se si cancella la frase "scatole di colore diverso contengono numeri diversi di biglie", cioè se rimane solo l'implicazione diretta "Se le scatole hanno lo stesso colore allora hanno lo stesso numero di biglie". Gli alunni scoprono che, in questo caso, in una situazione dove vi siano due scatole di colore diverso di cui si conosca il numero totale delle biglie, esse possono contenere comunque un numero uguale di biglie. Questo passaggio è importante ma è tutt'altro che banale sul piano didattico; presuppone un breve percorso di formazione per gli insegnanti sui nodi logici che stanno alla base delle regole $2 a$ e $2 b$, che dia loro delle pur embrionali competenze nel comprendere la differenza fra implicazione diretta e doppia implicazione.

8. Vedi Unità 6, Dalla bilancia a piatti all'equazione, 2003, p. 55
} 
Figura 3

Situazione problematica 1

Figura 4

Situazione problematica 2

Figura 5

Situazione problematica 3 .
(1)

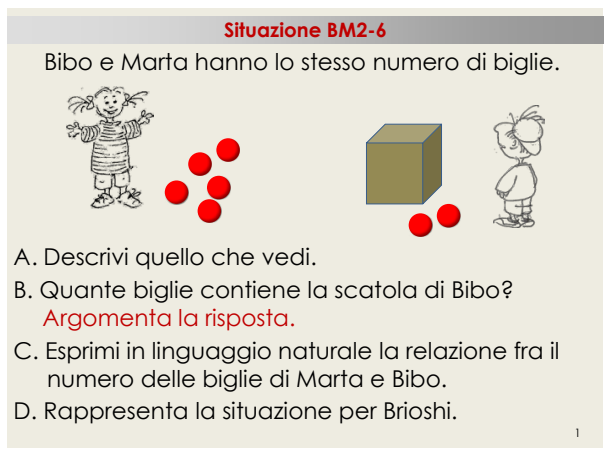

C. «ll numero delle biglie di Marta è uguale alla somma fra il numero delle biglie nella scatola e quello delle biglie di Bibo che si vedono».

D. $5=a+2$.

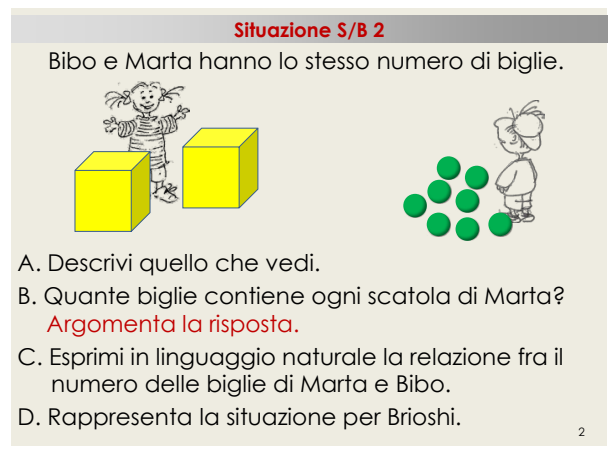

C. «ll doppio del numero delle biglie di una scatola è uguale al numero delle biglie di Bibo»;

D. Viene tradotta come $a+a=8$ e $a \times 2=8$. Si riflette su rappresentazioni additive e moltiplicative.

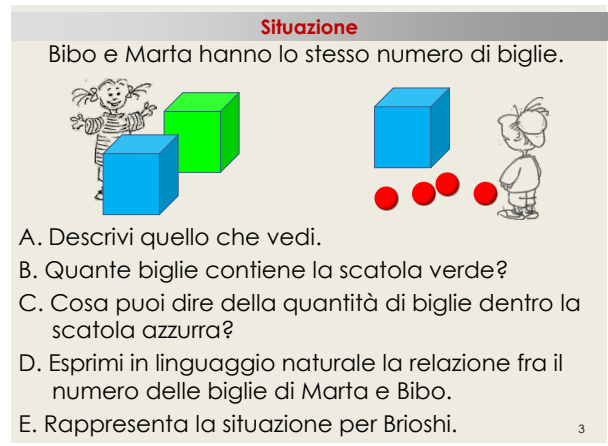

C. «La somma fra i numeri delle biglie nelle scatole di Marta è uguale alla somma fra il numero di biglie nella scatola e quello delle biglie fuori dalla scatola»;

D. $b+v=b+4$. Nel corso della discussione gli alunni capiscono che nella scatola verde ci sono 4 biglie e in quelle blu «c'è lo stesso numero ma non si può sapere quale».

Si propone poi una sfida che, sulla base di precedenti esperienze in altre classi, 
è considerata piuttosto complessa anche perché "rompe" con la prima regola del gioco che, come si vede nella parte alta della slide, questa volta viene posta in modo interrogativo:

(4)

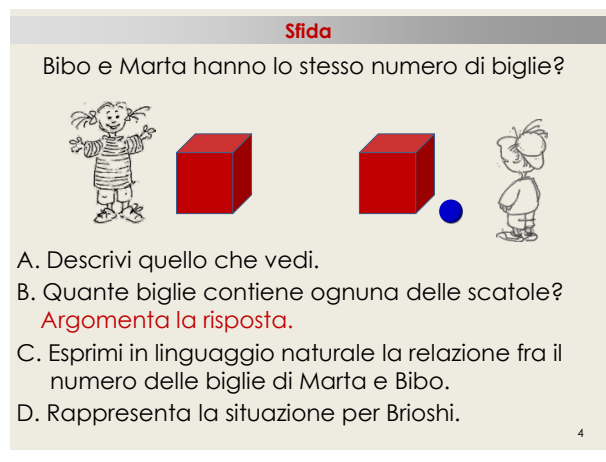

Al momento della risposta alla consegna D. 20 alunni su 24 scrivono nel giro di pochi secondi:

$a \neq a+1$.

L'insegnante partecipa da molti anni al progetto ArAl; i concetti chiave vengono affrontati e discussi nella classe all'interno di un contratto didattico che prevede anche una parziale, ma significativa, condivisione del quadro teorico con gli alunni. I principali concetti sono:

i. le diverse rappresentazioni di un numero, canonica / non canonica e la loro interpretazione; ad es. in (1) la scrittura a +2 viene descritta in termini relazionali come "somma fra il numero delle biglie nella scatola e quello delle biglie che si vedono";

ii. I'uguale come indicatore di equivalenza: nel concepire le rappresentazioni delle situazioni di Scatole \& Biglie gli alunni esprimono l'interiorizzazione del suo significato relazionale (v. par. 5.b.);

iii. la dualità rappresentare / risolvere: la classe dell'episodio rappresenta in modo trasparente le relazioni (di volta in volta additiva, moltiplicativa, di uguaglianza) fra gli enti in gioco. Alunni inesperti risolvono invece il problema eseguendo un'operazione; nella situazione (1), per esempio, rispondono semplicemente "3", limitandosi al risultato di un calcolo. Se si chiede loro di spiegarsi, rispondono: "Ho fatto 5 meno 2 ». Come si è già evidenziato, il concetto di rappresentazione di una situazione problematica è loro estraneo. Ci si può chiedere allora quale significato attribuiscano studenti di scuola media, che non abbiano mai incontrato l'early algebra, ad una frase come $5 \times h / 2=35$, in cui 5 sia la base di un triangolo e 35 l'area, nel momento in cui il loro imprinting li porta a vederla come "operazioni a sinistra e risultato a destra" piuttosto che come uguaglianza fra due rappresentazioni diverse dell'area di quel triangolo, espresse rispettivamente: a sinistra, come rappresentazione dell'area della classe dei triangoli di base 5 ed altezza indeterminata, definibile come "prodotto fra la base (5) e la metà dell'altezza" e, a destra, in forma canonica (35).

iv. la conquista della lettera, e quindi la possibilità di rappresentare mediante un simbolo un numero sconosciuto (come nelle prime tre situazioni), o un numero qualsiasi (come nella quarta). Gli alunni dell'episodio hanno incontrato l'incognita in prima elementare, attraverso attività e riflessioni alle quali fanno riferi- 
mento molte Unità della Collana ArAl; la lettera è I'ultimo passo di un lenta conquista che si evolve attraverso l'invenzione di altri simboli, metafore del numero sconosciuto: un fantasmino, una macchia, un punto interrogativo, un simbolo qualsiasi. La conquista graduale dei diversi significati che una lettera può assumere è un esempio di evoluzione del balbettio algebrico, (v. par. 2). La competenza nell'uso della lettera anche come variabile può portare una classe che, per esempio, esplori le piramidi (come quella esaminata nel par. 1.4.5.) ad esprimere in linguaggio matematico la regola «In una minipiramide il numero in alto è la somma dei numeri scritti nei mattoni alla base» in questo modo:

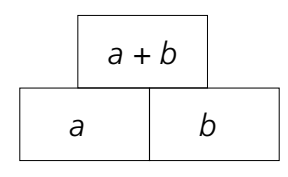

Questo passaggio ha implicazioni importanti sul piano matematico e linguistico man mano che aumenta il numero dei piani della piramide e gli alunni devono individuare una nuova regola (Cusi \& Navarra, 2012).

v. I'approccio al codice algebrico attraverso $\underline{B r i o s h i}{ }^{9}$, per il quale si chiede di rappresentare la situazione: Brioshi è un alunno virtuale giapponese, di età variabile a seconda di quella dei suoi interlocutori, che conosce solo la sua lingua ma sa usare molto bene il linguaggio matematico. Viene introdotto come "amico di penna matematica" per avvicinare alunni fra i 6 e i 14 anni alla semantica e alla sintassi del linguaggio matematico. Ad esempio, nel caso della situazione (2), nel corso della discussione attorno alle rappresentazioni individuali, gli alunni hanno dovuto confrontare, fra le altre, due tipi di rappresentazioni: $a \times 2=8 \mathrm{e}$ $8: 2=a$. Poiché la situazione è stata descritta come «ll doppio del numero delle biglie di una scatola di Marta è uguale al numero delle biglie di Bibo», hanno convenuto che sono entrambe corrette, ma che $a \times 2=8$ è la sua traduzione in linguaggio matematico, mentre $8: 2=$ a rappresenta l'operazione che permette di trovare il numero delle biglie di una scatola e i compagni che l'hanno proposta hanno lavorato nella prospettiva del risolvere, non del rappresentare. Nel primo caso $(a \times 2=8)$ sanno che Brioshi dovrebbe risolvere un'equazione (non la chiamano così ma le esperienze con la bilancia a piatti li hanno avvicinati agli embrioni di quelli che poi diverranno i principi di equivalenza), nel secondo $(8: 2=a)$ dovrebbe semplicemente eseguire la divisione $8: 2$, e sarebbe quindi un compito aritmetico, non algebrico. Questo gioco di ruolo funziona sempre, indipendentemente dall'età degli alunni, e Brioshi costituisce un richiamo forte alla correttezza e alla trasparenza delle scritture matematiche;

vi. interpretare in senso relazionale il significato di una scrittura in linguaggio matematico: nel corso dell'esplorazione della situazione (4) gli alunni (con sincero stupore dell'e-tutor, presente alla lezione) esprimono in termini relazionali i numeri di biglie dei due ragazzi, rispettivamente con a e $a+1$, e rappresentano con il simbolo $\neq$ la loro disuguaglianza; giustificano $a \neq a+1$, dicendo che «Bibo ha una biglia in più di Marta qualunque sia il numero di biglie nelle due scatole, che comunque è ogni volta uguale perché sono dello stesso colore». Rivolti all'insegnante osservano con aria sorniona «Volevi prenderci in giro ma non ci siamo

9. Vedi Unità 1, Brioshi e l'approccio al codice algebrico, 2003. 
cascati!». È interessante notare che in questo caso gli alunni non passano attraverso il linguaggio naturale, come hanno fatto nei tre casi precedenti, come traghetto semantico verso la rappresentazione in linguaggio algebrico, ma lo usano per spiegare, a posteriori, le ragioni della scrittura che hanno proposto. $\grave{E}$ importante inoltre anche il fatto che alunni di classi "esperte" abbiano capito che devono usare la stessa lettera per rappresentare il numero delle biglie in ognuna delle scatole di Bibo e Marta, numero che sapevano essere lo stesso.

\subsection{2 (P4, fine dell'anno scolastico)}

Figura 7

Situazione problematica 4

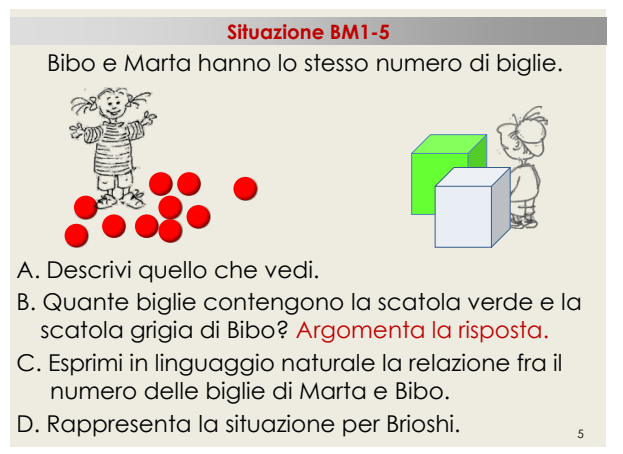

Anche questa classe è impegnata con una situazione problematica dell'attività Scatole \& Biglie; gli alunni lavorano seguendo le regole (2a) e (2b) (v. par. 6.1), che comprende la frase "scatole di colore diverso contengono numeri diversi di biglie", quindi non contemplano il caso 5-5.

Le argomentazioni relative al quesito B. sono collettive. Inizialmente gli alunni propongono in modo disordinato coppie pur corrette di numeri, poi qualcuno fa riferimento alla rappresentazione tabulare per organizzare una ricerca ordinata delle coppie di numeri che soddisfano alla condizione $10=a+b$ (si sa che una scatola può anche essere vuota):

(1)

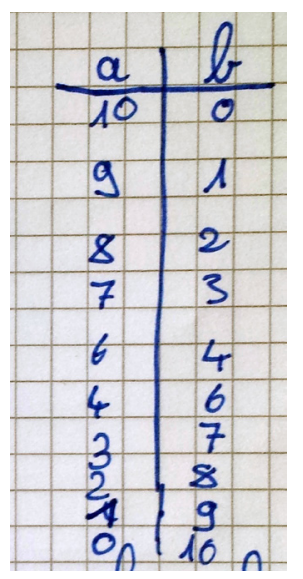

Dopo aver fatto analizzare alcune situazioni simili cambiando i numeri delle biglie di Marta, si chiede se sia possibile individuare una legge che permetta di stabilire quante sono le coppie di valori per un numero qualsiasi di biglie. Gli alunni lavorano individualmente. Le argomentazioni più complete sono simili e presentano delle sfumature attorno alle quali l'insegnante promuove la 
riflessione; due esempi:

(2)

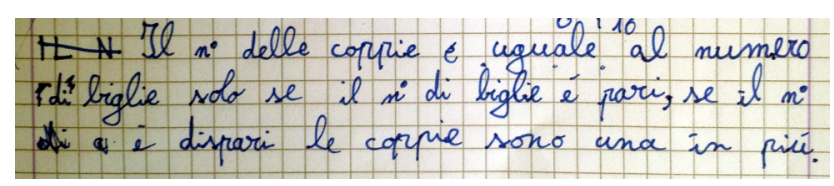

(3)

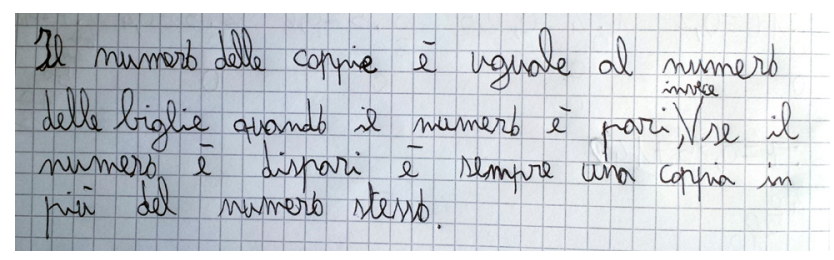

Si confrontano i termini "solo se" (2) e "quando" (1), si riflette sulla necessità o meno del termine "sempre" (2), si valuta se le parole "in più" acquistino maggiore chiarezza con la specificazione "del numero stesso" (3). Si conclude che le due frasi sono equivalenti.

La richiesta fatta alla classe - se sia possibile trovare una legge che permetta di stabilire quante sono le coppie per un numero qualsiasi di biglie - ha l'obiettivo di promuovere I'approccio alla generalizzazione attraverso la riflessione sulla struttura delle rappresentazioni in linguaggio matematico. Modificare il proprio insegnamento nella prospettiva dell'early algebra significa dare agli alunni l'opportunità di attivare differenti modi di pensare come: esplorare situazioni, analizzare relazioni tra quantità, prevedere, generalizzare, modellizzare, giustificare, verificare. Tale approccio è importante per promuovere lo sviluppo del pensiero algebrico sfruttando anche piccole opportunità che possono emergere in ambienti aritmetici e costituisce una preparazione fondamentale per il successivo studio dell'algebra (Cooper \& Warren, 2011). Impegnare alunni sin dalla scuola elementare in attività mirate alla generalizzazione è vitale, perché questo rinforza le loro competenze nel filtrare le informazioni matematiche in base alle loro caratteristiche comuni e nel progettare conclusioni in forma di rappresentazioni generali (Blanton \& al., 2018).

\subsection{3 ( $\mathrm{P} 4$, metà anno scolastico)}

Il terzo episodio si riferisce ad un problema del Rally Matematico Transalpino (RMT) interpretato e svolto collettivamente dagli alunni in chiave ArAl. Si tratta di un brano piuttosto lungo estratto da un diario (v. par. 3.1) in cui si è preferito mantenere la numerazione degli interventi per favorire la lettura dei commenti dell'insegnante (CI) e dell'e-tutor (C-ET) riferiti ad essi.

Andrea e Giacomo hanno da poco iniziato a collezionare figurine dei Pokemon. leri Andrea aveva 5 figurine in meno di Giacomo.

Oggi Giacomo ha ancora lo stesso numero di figurine che aveva ieri, invece Andrea ne ha ricevute in dono 21 e ora ha il doppio del numero di figurine di Giacomo.

Rappresenta la situazione in linguaggio matematico in modo che Brioshi possa trovare il numero delle figurine che ha oggi Andrea. 
L'insegnante (I) lancia una sfida:

1. I: «Potremmo trovare una soluzione pensando alla bilancia?»

2. Andrea: «Mettiamo da una parte le figurine di Giacomo e dall'altra quelle di Andrea».

3. Gaia: «Ma non sappiamo quante figurine ci sono in un piatto e quante nell'altro».

4. Anna: «Però sappiamo che Andrea aveva 5 figurine meno di Giacomo, quindi lui ne ha 5 in più».

5. Mayssa: «Sappiamo anche che alla fine Andrea avrà il doppio delle figurine di Giacomo».

6. Gaia: «I due piatti allora non sono in equilibrio!»

7. Francesco: «Quindi non si può usare la bilancia!»

8. Sebastiano: «Sì, c'è uno squilibrio!»

9. I: «Come possiamo rappresentarlo allora?» ${ }^{\text {c1-। }}$

C1-I: Il tentativo è di far sì che il problema sia affrontato non per tentativi ma con una modalità prealgebrica utilizzando i principi della bilancia (familiari agli alunni).

10. Adele: «lo pensavo di disegnare lo stesso la bilancia».

11. Mayssa: "All'inizio Giacomo ha più figurine quindi...».

12. Gaia: «Ma non sappiamo il numero di figurine che avevano all'inizio!»

13. Amel: «Allora usiamo una lettera».

14. Aurora: «Per esempio f di figurine». C2-ET

C2-ET: È sempre opportuno far esplicitare il significato delle lettere usate, per evitare che si formi il misconcetto che esse indichino persone, oggetti, colori e così via.

15. Amel: «ll piatto più pesante alla fine è quello di Andrea, il più leggero è quello di Giacomo». "3-Et

C3-ET: L'osservazione di Amel è corretta. Sarebbe stata opportuna una domanda che gli facesse esplicitare in base a quale informazione abbia ipotizzato che il piatto di Andrea è più pesante di quello di Giacomo. La risposta sarebbe stata importante per lui e per i compagni.

16. Mayssa: «Andrea ha il doppio delle figurine, ma 21 non si divide per due». ${ }^{\text {C4-Eт }}$

C4-ET: Mayssa non ha capito che Andrea oggi non ha solo 21 figurine (il numero dispari di cui parla), ma che ne ha 21 in più di quelle che aveva il giorno prima. Dario (rigo 19) supererà questo errore rappresentando il numero delle figurine di Andrea con " $f+21$ ".

17. Adele: "Se a Giacomo diamo il doppio delle figurine... (non sa come continuare)».

18. І: «Aiutate Adele in questo ragionamento».

19. Dario: «Prima, cioè ieri, la situazione era così (fa questo disegno alla LIM)»: ${ }^{C 5-E T}$

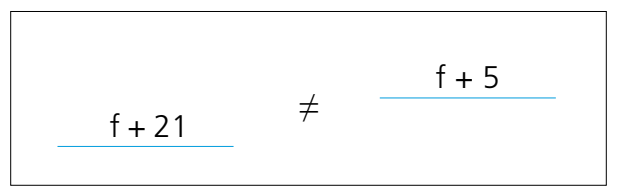

C5-ET: Ritengo opportuno chiarire che in realtà la "bilancia sbilanciata" di Dario 
non rappresenta "la situazione di ieri" come lui dice (rigo 19), ma il confronto fra la situazione di oggi (a sinistra) e quella di ieri (a destra); indica con:

- " $f$ " il numero delle figurine di Andrea;

- " $f+5$ " quello delle figurine che Giacomo aveva ieri (5 in più di quelle di Andrea);

- " $f+21$ " quello delle figurine che ha oggi Andrea (21 in più di quelle che lui stesso aveva ieri).

20. I: «Quindi per trovare un equilibrio come diceva Gaia?»

21. Anna: «Giacomo deve avere il doppio delle figurine di Andrea».

22. Mayssa scrive alla LIM: $f+21=f+5 \times 2$.

23. Sebastiano: «Devi mettere le parentesi».

24. Mayssa scrive: $f+21=(f+5) \times 2$.

25. I: «Bravissimi!!! E adesso? Sul piatto a destra devo applicare...».

26. Alcuni: «... la proprietà distributiva!»

27. Sebastiano: «Ah, sì!!! Allora diventa $2 \times f+2 \times 5 \ldots$ ».

28. Anna: "Quindi sarebbe...» viene a scrivere alla LIM: $f+21=2 \times f+10$.

29. Mayssa: «Tolgo una $f$ da entrambe le parti della bilancia così la bilancia resta in equilibrio, è il primo principio della bilancia!» Assieme ad Anna scrive: $f+21-f=2 \times f-f+10$.

30. Anna, che è ancora alla LIM, scrive nella riga sottostante ciò che rimane: $21=f+10$.

31. Francesco: «f è $11 »$.

32. I: «Non dovete risolvere, rappresentate bene per Brioshi quello che fate».

33. Amel: «21 è come $11+10 »$.

34. Anna: «È una forma non canonica di $21 »$.

35. Adele scrive $11+10=f+10$ : «Adesso tolgo 10 di qua e 10 di là».

36. Gaia: «Sì, è il primo principio della bilancia!!! E rimane $f=11$. Ci siamo riusciti!!!»

37. Sebastiano: «Infatti $11+21=32 \ldots$ è il numero di figurine di Andrea!»

38. Anna: «E Giacomo ne ha la metà... 16!»

Ritengo che il diario testimoni i livelli ai quali può arrivare una quarta elementare che dalla prima abbia lavorato in modo coerente e continuativo nella prospettiva dell'early algebra. L'insegnante interviene lo stretto necessario, favorendo la devoluzione e lo scambio fra pari; gli alunni intrecciano in modo positivo i ragionamenti evidenziando, attraverso l'uso della lettera, un controllo consapevole sul significato di termini come "doppio" e "metà", di un segno inconsueto come " $\neq$ ", di una proprietà altrettanto poco frequentata come la distributiva, ${ }^{10}$ della bilancia e dei suoi principi come approccio all'equazione e alla sua soluzione, ${ }^{11}$ degli aspetti semantici e sintattici del linguaggio matematico approfonditi attraverso il "mediatore culturale matematico" Brioshi, di costrutti come "forma non canonica", di verifica finale dei risultati ottenuti. Riemergono alcuni fra i principali temi evidenziati in precedenza, supportati da aspetti metodologici altrettanto importanti, attinenti il piano sociale e quello linguistico: una conduzione da parte dell'insegnante rispettosa delle modalità più produttive per condurre in modo efficace una discussione di classe su temi ma-

10. Vedi Unità 11, Viaggio alla conquista della proprietà distributiva, 2008.

11. Vedi Unità 5, Le piramidi di numeri, 2003. 
tematici, I'abitudine alla verbalizzazione e all'argomentazione da parte degli alunni, la chiarezza del contratto didattico al fine di attivare la costruzione sociale della conoscenza, la condivisione del quadro teorico con gli alunni, che divengono così non più soltanto consumatori ma produttori di pensiero matematico. Tutto questo, nella cornice di un problema "intelligente", proveniente da quella prodigiosa fucina che è RMT che fra l'altro affonda una parte delle sue radici, come il progetto ArAl, nell'importante storia dei Nuclei di ricerca italiani in didattica della matematica.

\title{
7 Rappresentare vs risolvere un problema
}

Consideriamo un ultimo tema, cruciale per l'evoluzione del balbettio algebrico: i problemi.

I problemi che tradizionalmente affrontano gli alunni della scuola italiana, classificabili come problemi verbali standard, sono ben diversi dalle situazioni problematiche come quelle del RMT e producono nell'immaginario della maggior parte delle persone, com'è noto, la convinzione che risolvere problemi significhi individuare le operazioni che permettono di trovare il risultato agendo sui dati. Vediamo quindi come cambia la situazione se si modifica il punto di vista e si sostituisce quello aritmetico del riso/vere con quello algebrico del rappresentare.

Consideriamo un esempio tratto da un sussidiario di IV elementare:

\begin{abstract}
Alle gare provinciali studentesche di atletica partecipano 19 scuole, ognuna delle quali invia una squadra formata da 22 atleti. Alla fine di tutte le altre gare iniziano le batterie dei centometristi, alle quali partecipano 54 studenti.

Quanti studenti hanno partecipato alle gare precedenti le batterie dei centometristi?
\end{abstract}

Generalmente un alunno lo risolve con due operazioni distinte, $22 \times 19=418 \mathrm{e}$ $418-54=364$ : opera cioè su tre enti $(22,19,54)$ e su un quarto ente intermedio (418) mediante due operazioni: una moltiplicazione e una sottrazione; il risultato finale fornisce la risposta alla domanda del problema.

Manteniamo lo stesso testo, ma impostiamo la consegna nella prospettiva del rappresentare:

Rappresenta la situazione in modo che Brioshi trovi quanti studenti hanno partecipato alle gare precedenti le batterie dei centometristi.

La conseguenza del cambio della consegna è che non è più lui, lo studente, il risolutore; egli deve preparare una scrittura che permetta ad un altro (a Brioshi, o ad un compagno, o ad un'altra classe) di risolvere il problema. Avviene una sorta di rivoluzione copernicana; l'alunno:

a. non tiene conto più, come prima, di tre dati (cioè dei numeri conosciuti) ma di quattro enti: il numero delle scuole (19), quello degli atleti di ogni squadra (22), quello degli atleti che partecipano alle gare dei centometristi (54) e quello sconosciuto degli studenti che hanno gareggiato sino a quel momento (che decide di indicare, per esempio, con s); 
b. non individua più due operazioni (una moltiplicazione e una sottrazione da svolgere sequenzialmente) ma tre relazioni fra i quattro enti: una moltiplicativa fra il numero delle scuole (19) e quello degli atleti di ogni squadra (22), una additiva (espressa attraverso una sottrazione) fra il numero di tutti gli atleti (espresso dal prodotto tra 22 e 19) e il numero dei centometristi e infine un'uguaglianza.

L'alunno quindi interpreta la situazione problematica ed esprime non come si trova, ma cos'è il numero degli studenti che hanno partecipato alle gare precedenti le batterie dei centometristi, e lo esprime in linguaggio naturale attraverso una definizione relazionale: "Il numero degli studenti che hanno già fatto le loro gare è uguale alla differenza fra il numero di tutti gli atleti, che è il prodotto fra 22 e 19, e il numero dei centometristi, 54". Traduce quindi in questo modo: $g=22 \times 19-54$, permettendo così a Brioshi (che in questo 'gioco di ruolo' sono gli stessi alunni) di trovare il valore di $g$ (364). Il cambio della consegna ha, in una prima fase, lo scopo di far cogliere a docenti e alunni la differenza fra risolvere un problema e rappresentarlo. Una volta risolto il problema, questo spostamento di attenzione consente di oggettivare la sua struttura: gli alunni, esplorando la scrittura iniziale $g=22 \times 19-54$, vengono guidati verso la scoperta che essa è la particolarizzazione di una formula del tipo generale, in cui $i$ sia il numero degli atleti per ogni istituto, s il numero delle scuole partecipanti, $c$ il numero dei centometristi, $g$ il numero degli studenti che partecipano alle altre gare, che può essere scritta per esempio in questo modo: $g=i \times s-c$. L'insegnante può inoltre chiedere di inventare altre situazioni, in contesti diversi, rappresentabili con la medesima frase.

Vediamo infine come si potrebbe modificare il testo dello stesso problema in modo da renderlo davvero significativo sul piano algebrico, trasformando in incognita uno dei dati interni al testo. L'obiettivo in questo caso è quello di guidare gli alunni verso una rappresentazione che costituisca per Brioshi un'equazione da risolvere:

\footnotetext{
Alle gare provinciali studentesche di atletica partecipano alcune scuole, ognuna delle quali invia una squadra formata da 22 atleti. Nella prima parte della giornata gareggiano 364 atleti; infine iniziano le batterie dei centometristi, alle quali partecipano 54 studenti.

Rappresenta la situazione in modo che Brioshi trovi quante scuole hanno partecipato alle gare.
}

Attraverso il confronto tra le rappresentazioni in linguaggio naturale della situazione, prodotte individualmente o in gruppi, gli alunni giungono ad una formulazione condivisa, per esempio: "Il prodotto fra il numero degli atleti inviati da ogni scuola (22) e il numero delle scuole (per esempio s) è uguale alla somma fra il numero degli atleti che partecipano alle gare precedenti (364) e il numero dei centometristi (54)". La traduzione (anche in questo caso letterale) è: $22 \times s=364+54$. Ora sì che Brioshi (gli alunni stessi) deve attivare le sue conoscenze acquisite attraverso la bilancia a piatti, e quindi applicare dove necessario quelli che sono stati chiamati "primo e secondo principio della bilancia" (cioè i principi di equivalenza); un protocollo scritto da alunni in questo caso è il seguente (sanno di dover illustrare ogni passaggio):

$22 \times s=364+54$

$22 \times s=418 \quad$ sostituzione della forma non canonica $364+54$ con la canonica 418

$22 \times s: 22=418: 22$ secondo principio (divido per 22 da entrambe le parti)

$s=19 \quad$ numero delle scuole che hanno partecipato alle gare. 
Ma questa che abbiamo analizzato è solo una delle rappresentazioni possibili. Alcuni alunni potrebbero interpretare il problema da un altro punto di vista: "II numero delle scuole che hanno partecipato alle gare $(s)$ è il quoziente fra la somma degli atleti ( $364+54$ ) e quello degli alunni di ogni scuola (22)". La rappresentazione in linguaggio matematico sarebbe: $s=(364+54): 22$.

L'insegnante può proporre ora il confronto fra $22 \times s=364+54$ e $s=(364+54): 22$, aprendo così alla riflessione sulle parafrasi (v. par. 4.1.7.), e cioè sulla struttura di rappresentazioni diverse della stessa situazione in cui sia cambiato il punto di vista del risolutore. Ma può andare oltre, proponendo un ulteriore confronto con le operazioni eseguite per risolvere la prima versione del problema $(22 \times 19=418$ e $418-54=364)$. Se gli alunni riscrivono le operazioni staccate mediante un'unica rappresentazione in cui il risultato (364) venga rappresentato con un'incognita ottengono una nuova parafrasi, $22 \times 19-54=9$, che potrebbe essere tradotta in linguaggio naturale: "La differenza fra il prodotto del numero degli alunni per scuola (22) con il numero delle scuole (19) e il numero dei centometristi è uguale al numero degli studenti che hanno partecipato alle gare precedenti $(g)^{\prime \prime}$.

\section{Conclusioni}

Portare alunni dai 5 ai 14 anni all'incontro con l'early algebra significa essenzialmente guidarli - attraverso situazioni problematiche create appositamente e affrontate in modo socio-costruttivo - verso un nuovo linguaggio, con la sua semantica e la sua sintassi. Le radici epistemologiche del progetto ArAl sono intimamente connesse con un approccio linguistico; le discipline dell'aritmetica e dell'algebra sono viste come unite in una meta-disciplina dotata di uno specifico linguaggio unificatore. Il rispetto delle regole di questo linguaggio diventa essenziale per poter svolgere attività quali la traduzione, la discussione, l'interpretazione, la previsione e la comunicazione, concepite come attività matematiche. L'effettuare calcoli è presente ma è subordinato a scopi più alti: il ragionamento, le argomentazioni, le confutazioni, le correzioni. Man mano che l'algebra affrontata dagli alunni cresce in complessità, essi saranno guidati a comprendere che la manipolazione delle forme simboliche non sia fine a se stessa, ma aiuti a matematizzare, esplorare, dedurre, raggiungere nuove conoscenze.

Alcuni degli aspetti educativi che ho descritto vanno sviluppati costantemente negli alunni, poiché supportano in loro la crescita del pensiero algebrico. Parlo della riflessione: (i) sul linguaggio per favorire la capacità di costruire argomentazioni, tradurre dal linguaggio naturale al linguaggio algebrico e viceversa e produrre pensieri originali; (ii) sulla relazione tra intuizioni o produzioni individuali e la loro condivisione, in modo da promuovere la costruzione sociale della conoscenza; (iii) sul passaggio da situazioni generative concrete alla condensazione concettuale dei fatti matematici sottostanti e alla loro rappresentazione; (iv) su aspetti matematici di base come il riconoscimento dell'equivalenza strutturale tra frasi o, nel caso di dati sconosciuti o variabili, la generazione di equazioni e funzioni.

Riteniamo che gli alunni possano sviluppare il pensiero algebrico purché vengano educati come studenti metacognitivi e per questo è necessario che gli insegnanti, a loro volta, diventino insegnanti metacognitivi. Per promuovere la metacognizione, 
dal 2001 elaboriamo strumenti e strategie anche attraverso il coinvolgimento costante degli stessi docenti, in uno stretto intreccio di riflessioni sulla conoscenza in gioco (teoria) e sull'azione in classe (pratica): la Collana ArAl, i materiali visionabili nel sito http://www.progettoaral.it, le collaborazioni annuali con istituti o reti di istituti, i commenti ai diari, gli scambi via mail con i docenti, il gruppo 'Progetto ArAl' in Facebook.

La nostra esperienza e i nostri studi di ricerca ci hanno resi consapevoli che cambiare I'insegnamento nella prospettiva dell'early algebra richiede una conversione della professionalità degli insegnanti, costruita sulla rilettura in chiave critica di conoscenze, convinzioni, atteggiamenti, modalità di conduzione delle attività, stereotipi (forse) e sulla presa di coscienza del fatto che l'aritmetica e l'algebra vanno considerate come discipline intrecciate fin dall'inizio della scuola elementare. Questo processo è lento e deve essere sostenuto attraverso appropriati programmi di sviluppo. Per mantenere viva questa prospettiva nell'attività in classe gli insegnanti devono affinare la loro sensibilità per riconoscere le continue micro-situazioni in cui sia possibile porre a confronto il punto di vista operativo proprio e degli alunni con quello relazionale. Per raggiungere questo obiettivo e costruire gradualmente e consapevolmente abilità matematiche, è necessario che alunni e insegnanti condividano termini specifici del quadro teorico dell'early algebra, usandoli costantemente quando discutono, riflettendo sul loro significato e facendo emergere le loro connessioni. Crediamo che su questa base gli alunni possano sperimentare, sin dai primi anni di scuola, un approccio consapevole al linguaggio e al pensiero algebrico e, in generale, un atteggiamento positivo nei confronti della matematica.

\section{Bibliografia}

AA.VV. (2003-2018). Progetto ArAl: Percorsi nell'aritmetica per favorire il pensiero prealge$\underline{\text { brico. }}$. Collana (Quadro teorico e Glossario + 13 Unità). Bologna: Pitagora.

Blanton, M., Brizuela, B.M., Stephens, A., Knuth, E., Isler, I., Murphy Gardiner, A., Stroud, R., Fonger, N.L., \& Stylianou, D. (2018). Implementing a Framework for Early Algebra. In C. Kieran (Ed.), Teaching and Learning Algebraic Thinking with 5-to 12-Year-Olds, The Global Evolution of an Emerging Field of Research and Practice (pp. 27-50). Springer. ISBN 978-3-319-68350-8.

Carpenter, T.P., Franke, M.L., \& Levi, L. (2003). Thinking Mathematically. Integrating Arithmetic and Algebra in the Elementary School. Portsmouth, $\mathrm{NH}$ : Heinemann.

Carraher, D. W., Schliemann, A. D., \& Schwartz, J. L. (2007). Early algebra is not the same as algebra early. In J. Kaput, D. Carraher \& M. Blanton (Eds.), Algebra in the Early Grades (pp. 235-272). Mahwah, NJ: Erlbaum.

Carraher, D. W., \& Schliemann, A. D. (2018). Cultivating Early Algebra Thinking. In C. Kieran (Ed.), Teaching and Learning Algebraic Thinking with 5-to 12-Year-Olds, The Global Evolution of an Emerging Field of Research and Practice (pp. 107-138). Springer. ISBN 978-3319-68350-8.

Cooper, T.J., \& Warren, E. (2011). Years 2 to 6 students' ability to generalize: models, representations and theory for teaching and learning. In J. Cai \& E. Knuth (Eds.), Early algebraization, A Global Dialogue from Multiple Perspectives (pp. 483-510). Advances in Mathematics Education: Springer. 
Cusi, A., Malara, N.A., \& Navarra, G. (2011). Early Algebra: Theoretical Issues and Educational Strategies for Promoting a Linguistic and Metacognitive Approach to the Teaching and Learning of Mathematics. In J. Cai \& E. Knuth (Eds.), Early algebraization: A Global Dialogue from Multiple Perspectives (pp. 483-510). Advances in Mathematics Education. Berlin: Springer.

Cusi, A., \& Navarra, G. (2012). Aspetti di generalizzazione con alunni giovani in ambiente early algebra. Versione italiana di: Cusi, A., \& Navarra, G. (2012). Aspects of Generalization in Early algebra. In M. Tatsis \& K. Tatsis (Eds.), Generalization in mathematics at all educational levels (pp. 182-192). Rzeszòw University press, Rzeszòw (Poland). ISBN 978 83-7338-780-5.

Deon, V., \& Navarra, G. (2014). Come parlano gli insegnanti? In A. Colombo \& G. Pallotti (A cura di), L'italiano per capire (pp. 243-255). I Quaderni del GISCEL. Reggio Emilia. Aracne Editrice. ISBN 978-88-548-6817-5.

Kieran, C., Pang J., Schifter D., \& Ng, S. (2016). Early Algebra, Research into its Nature, its Learning, its Teaching. ICME 13 Topical Survey. Springer. ISBN 978-3-319-32258-2.

Malara, N.A. (1994). II pensiero algebrico: come promuoverlo sin dalla scuola dell'obbligo limitandone le difficoltà? In B. D'Amore (A cura di), L'apprendimento della matematica: dalla Ricerca Teorica alla Pratica Didattica (pp. 67-77). Bologna: Pitagora.

Malara, N.A., \& Navarra, G. (2018). New words and concepts for early algebra teaching: sharing with teachers epistemological issues in early algebra to develop students' early algebra thinking. In C. Kieran (Ed.), Teaching and Learning Algebraic Thinking with 5-to 12-Year-Olds, The Global Evolution of an Emerging Field of Research and Practice (pp. 51-78). Springer. ISBN 978-3-319-68350-8.

Mason, J. (2002). Researching Your Own Practice: the Discipline of Noticing. London: The Falmer Press.

Navarra, G. (2009). Early algebra: un approccio relazionale all'aritmetica per promuovere una concezione linguistica dell'algebra. In P. Baratter \& S. Dallabrida (A cura di), Atti del Convegno GISCEL: Lingua e grammatica, Teoria e prospettive didattiche (pp. 133-154). Milano: Franco Angeli.

Navarra, G. (2016). "Cinque per tre fa quin... ?" "... dici" "Bravo!" La metodologia delle trascrizioni pluricommentate come strumento per lo studio dei comportamenti linguistici dei docenti di matematica e la promozione di sensibilità e competenze in tale ambito. In F. De Renzo \& M.E. Piemontese (A cura di), Atti del Convegno Nazionale GISCEL 2014 (pp. 239-252). Roma.

Steinweg, A.S., Akinwunmi, K., \& Lenz, D. (2018). Making Implicit Algebraic Thinking Explicit: Exploiting National Characteristics of German Approaches. In C. Kieran (Ed.), Teaching and Learning Algebraic Thinking with 5-to 12-Year-Olds, The Global Evolution of an Emerging Field of Research and Practice (pp. 283-307). Springer. ISBN 978-3-319-68350-8.

\author{
Autore/Giancarlo Navarra \\ Insegnante ricercatore, già professore a contratto - Università di Modena e Reggio \\ Emilia, Italia \\ giancarlonavarra@gmail.com
}

